\title{
A preliminary investigation of the responsiveness or otherwise of patients and staff of a leprosy hospital to groups of shared or species specific antigens of mycobacteria
}

\author{
J L STANFORD, $*$ P M NYE, $*$ G A W ROOK, ${ }^{*}$ N SAMUEL ${ }^{\dagger} \&$ \\ A FAIRBANK $\dagger$ \\ *School of Pathology, Middlesex Hospital Medical School, \\ Riding House Street, London W1P $7 L D$, and $\dagger$ Anandaban \\ Leprosy Hospital, PO Box 151, Kathmandu, Nepal
}

Received for publication 31 March 1981

\begin{abstract}
Summary In an attempt to classify skin-test responsiveness of leprosy patients according to the groups of antigens, rather than the individual mycobacterial species to which they respond, we have tested patients and staff members at Anandaban leprosy hospital in Nepal with Burulin (made from Mycobacteria ulcerans) and 3 specially mixed reagents. Ability to make a positive response to group i, common mycobacterial antigens, was almost absent and to group ii, antigens associated with slow growers, was markedly impaired in the patient groups. However, positive responses to group iv, species specific, antigens of slowly growing species were retained. Non-specific skin-test unresponsiveness (Category 2) due either to sequestration of the relevant cells outside the circulation or to circulating suppressor factors was present in 2 out of 27 staff members, 9 out of 24 TT/BT patients, 11 out of $18 \mathrm{BL}$ patients and 10 out of $22 \mathrm{LL}$ patients. Evidence of a suppressor mechanism possibly triggered by group iv antigens of fast growers and operative on positive responses to slow growers, was demonstrable in 3 out of 12 staff members, 8 out of 14 TT/BT patients, 7 out of $7 \mathrm{BL}$ patients and 6 out of 12 LL patients.

It cannot at the moment be proved whether these observations are related to susceptibility to the disease, or are consequences of it. However, the presence of the same, or similar, suppressory phenomena amongst staff members argues against the latter.
\end{abstract}

\section{Introduction}

The soluble antigens present in sonicate preparations of mycobacteria which are demonstrable in immunodiffusion analysis with hyperimmune rabbit anti- 
sera can be divided into 4 groups. ${ }^{1}$ Group i consists of antigens common to all species of mycobacteria and nocardiae. Group ii antigens are shared by slowly growing species and absent from rapid growers. Group iii antigens are shared by most rapidly growing species and absent from slow growers. Group iv consists of those antigens limited to individual species. We have prepared 4 reagents with which to attempt to assess the part these groups of antigens may play in skin-test reactivity in leprosy patients. The results of a preliminary investigation of their use are presented.

\section{Materials and methods}

\section{REAGENTS}

These were prepared from the stock concentrates of our New Tuberculins: ${ }^{2}$

1. Pooled slow grower reagent (SG). Equal volumes of filter sterilized sonicate preparation of 12 different slowly growing mycobacteria were pooled. Each reagent used contained $1 \mathrm{mg}$ of protein $/ \mathrm{ml}$.

2. Pooled fast grower reagent $(\mathrm{FG})$. This was produced as described above from sonicates of 12 fast growers, again each at a concentrate of $1 \mathrm{mg}$ $\operatorname{protein} / \mathrm{ml}$.

3. Fast grower/slow grower mixtures $(\mathrm{F} / \mathrm{S})$. This was prepared by mixing equal volumes of SG and FG (see Table 1).

For use each reagent was diluted in borate buffer at $\mathrm{pH} 8.0\left(\mathrm{Na}_{2} \mathrm{~B}_{4} \mathrm{O}_{7}\right.$. $10 \mathrm{H}_{2} \mathrm{O}, 3.36 \mathrm{~g} ; \mathrm{H}_{3} \mathrm{BO}_{3}, 5.25 \mathrm{~g} ; \mathrm{NaCl}, 6.19 \mathrm{~g}$; Tween $80,0.0005 \%$, distilled water to 1 litre) to a concentration of $2 \mu \mathrm{g}$ protein $/ \mathrm{ml}$, and dispensed into $5 \mathrm{ml}$ tuberculin vials through a sterile $0.2 \mu \mathrm{m}$ membrane filter.

4. Burulin (B). This was our standard new tuberculin preparation from $M$. ulcerans, ${ }^{3}$ dispensed as described above at a concentration of $2 \mu \mathrm{g}$ protein $/ \mathrm{ml}$.

Table 1. Contents of the reagents mixtures

\begin{tabular}{ll}
\hline \multicolumn{1}{c}{ FG } & \multicolumn{1}{c}{ SG } \\
\hline Chitin & Aviumin A \\
Diernhoferin & Aviumin B \\
Duvalin & Aviumin C \\
Flavescin & A*-in \\
Gilvin & Burulin \\
Neoaurumin & Gordonin \\
Nonchromogenicin R507R & Kansasin 8 \\
Nonchromogenicin R812R & Kansasin 1129 \\
Ranin & Marinin \\
Rhodesin & Scrofulin \\
Vaccin R859R & 'Talwin' \\
Vaccin R877R & Xenopin \\
\hline
\end{tabular}


$0.1 \mathrm{ml}$ volumes of the 4 reagents were injected simultaneously, 2 on each forearm, on groups of staff members and patients at Anandaban leprosy hospital in Nepal. Diameters of induration were measured after 72 hours, a mean diameter of induration of $2 \mathrm{~mm}$ or more was taken as positive. Twentyseven staff members and 64 patients agreed to take part.

\section{Results}

Percentages of positive responses to each reagent and mean positive reaction sizes in each of the groups tested are shown in Table 2. Within each of the patient groups responses were fewer and, in general, smaller than in the staff group. Six staff members, $1 \mathrm{BT}, 4 \mathrm{BL}$ and $2 \mathrm{LL}$ patients had $\mathrm{BCG}$ vaccination scars, but these persons did not seem to have any particular pattern of response.

Table 2. Numbers tested in each study group, numbers and percentages of persons producing positive responses within each group, and mean positive reaction sizes

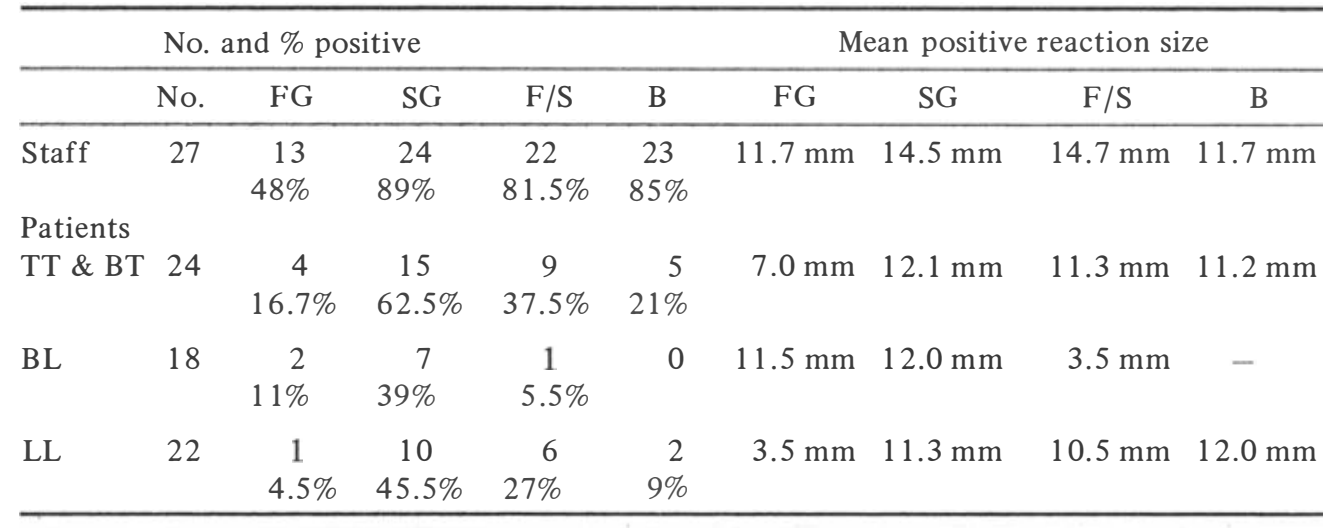

Thirteen $(48 \%)$ of the staff members responded positively to all 4 reagents with a mean response size of $13.3 \mathrm{~mm}$ (range $3.5-25 \mathrm{~mm}$ ) and $2(7.4 \%)$ of the staff were negative to all reagents. Amongst tuberculoid (TT) and borderline tuberculoid (BT) patients one $(4.2 \%)$ was positive to all reagents and $9(37.5 \%)$ were negative to all reagents. Of the borderline lepromatous (BL) and lepromatous (LL) patients none were positive to all 4 reagents and $11(61 \%)$ and $10(45.5 \%)$, respectively, were negative to all reagents. The results for those individuals who responded positively to 1 or more, but not all, reagents are shown in Table 3.

\section{Discussion}

Previous studies ${ }^{4}$ have shown that multiple skin-testing divides any population into three categories. Category 1 persons respond to all new tuberculins even 
Table 3. Individual skin test responses expressed as mean diameters of induration in $\mathrm{mm}$ in persons of Category 3

\begin{tabular}{|c|c|c|c|c|c|c|c|c|c|c|c|}
\hline & $\begin{array}{l}\text { Individual } \\
\text { No. }\end{array}$ & FG & SG & $\mathrm{F} / \mathrm{S}$ & B & & $\begin{array}{c}\text { Individual } \\
\text { No. }\end{array}$ & $\mathrm{FG}$ & SG & $\mathrm{F} / \mathrm{S}$ & B \\
\hline \multirow[t]{14}{*}{ Staff } & 3 & 0 & 12 & 20 & 0 & BL & 2 & 0 & 8 & 0 & 0 \\
\hline & 5 & 0 & 16 & 15 & 12 & & 3 & 0 & 17 & 0 & 0 \\
\hline & 8 & 0 & 26 & 19 & 16 & & 4 & 0 & 9 & 0 & 0 \\
\hline & 9 & 0 & 15 & 0 & 14 & & 6 & 0 & 9 & 0 & 0 \\
\hline & 11 & 0 & 8 & 0 & 9 & & 12 & 13 & 13 & 0 & $0^{*}$ \\
\hline & 12 & 0 & 0 & 16 & 0* & & 16 & 8 & 18 & 0 & $0^{*}$ \\
\hline & 13 & 0 & 15 & 10 & 8 & & 18 & 0 & 8 & 4 & 0 \\
\hline & 15 & 0 & 13 & 0 & 11 & & & & & & \\
\hline & 31 & 0 & 13 & 13 & 15 & & & & & & \\
\hline & 34 & 0 & 17 & 14 & 13 & LL & 3 & 0 & 0 & 0 & $12^{*}$ \\
\hline & 35 & 0 & 12 & 14 & 4 & & 5 & 0 & 15 & 8 & 0 \\
\hline & 36 & 0 & 12 & 11 & 9 & & 6 & 0 & 14 & 0 & 0 \\
\hline & & & & & & & 7 & 0 & 15 & 0 & 0 \\
\hline & & & & & & & 16 & 0 & 5 & 0 & 0 \\
\hline \multirow[t]{11}{*}{ BT } & 1 & 0 & 9 & 0 & 0 & & 17 & 0 & 11 & 0 & 0 \\
\hline & 6 & 4 & 13 & 7 & 0 & & 19 & 0 & 16 & 14 & 0 \\
\hline & 7 & 0 & 10 & 15 & 0 & & 20 & 0 & 13 & 9 & 12 \\
\hline & 8 & 0 & 9 & 0 & 0 & & 22 & 0 & 10 & 0 & 0 \\
\hline & 9 & 0 & 12 & 0 & 0 & & 23 & 0 & 7 & 9 & 0 \\
\hline & 14 & 0 & 8 & 10 & 9 & & 24 & 0 & 0 & 11 & $0^{*}$ \\
\hline & 15 & 10 & 12 & 14 & 0 & & 25 & 4 & 8 & 9 & 0 \\
\hline & 18 & 0 & 8 & 0 & 0 & & & & & & \\
\hline & 27 & 0 & 11 & 0 & 4 & & & & & & \\
\hline & 28 & 0 & 14 & 0 & 0 & & & & & & \\
\hline & 30 & 4 & 11 & 5 & 0 & & & & & & \\
\hline \multirow[t]{3}{*}{$\mathrm{TT}$} & 2 & 0 & 20 & 6 & 0 & & & & & & \\
\hline & 4 & 0 & 11 & 16 & 0 & & & & & & \\
\hline & 6 & 0 & 17 & 12 & 18 & & & & & & \\
\hline
\end{tabular}

those prepared from species unlikely to have been met. For example, Mycobacterium leprae in Great Britain and M. ulcerans in Nepal. Thus these individuals are responding to common (probably group i) antigens or combinations of these and species specific (group iv) antigens of those species that have been met. Category 2 persons fail to respond to the normal test dose $(0.2 \mu \mathrm{g}$ protein) of any of the new tuberculins even though many of the species used to prepare them will have been met and perhaps transitorily responded to in the past. For example, such persons are usually temporarily positive responders to Tuberculin after BCG vaccination. Category 2 individuals either have sequestration of competent lymphocytes outside of the free circulation, perhaps in the spleen or lymph nodes, or have a cell mediated suppressor mechanism triggered by group i antigen or a circulating blocking substance, perhaps an antibody. The third and largest category is of persons responding positively to some new tuberculins and not to others. Such persons respond to group iv antigens of 
the species they have met in most cases and perhaps sometimes to group ii antigen.

Reagent SG in this study contains the normal concentrations of groups $\mathrm{i}$ and ii and twelvefold dilutions of each of the group iv antigens of the organisms included. Similarly the FG reagent contains normal concentrations of groups i and iii antigen and a twelvefold dilution of the group iv antigens of the fast growing organisms. The combined reagent $\mathrm{F} / \mathrm{S}$ contains the normal concentration of group i antigen, half concentrations of groups ii and iii antigen and twenty-fourfold dilutions of all the group iv antigens. The single reagent Burulin contains approximately normal concentrations of groups i and ii antigen plus the group iv antigens of $M$. ulcerans that none of our study group should have met. It should be pointed out as a rough rule of thumb, that tenfold changes in reagent concentration produce approximately twofold changes in diameters of induration in positive responses.

Figure 1 shows the apportionment to the different categories of responders achieved in our study groups. Except for one person out of 24 in the TT/BT group, none of the patients belong to Category 1; thus it can be concluded that the ability to produce a positive response to group $\mathrm{i}$ antigen is almost

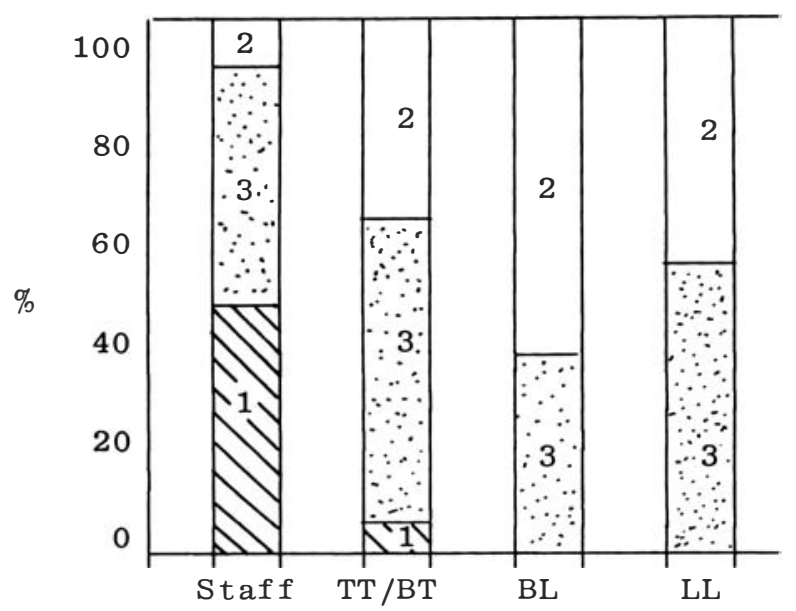

Figure 1. Division of the study groups into the categories of skin test responsiveness.

completely suppressed or absent in leprosy patients. ${ }^{4}$ Conversely, Category 2 is markedly expanded in each of the disease categories, in principle supporting previous observations. ${ }^{5}$ This non-specific unresponsiveness to mycobacterial antigens in patients with a mycobacteriosis has to be due to either active suppression or the absence of circulating clones of relevant lymphocytes.

The expansion of Category 3 to show the responses of each individual in Table 3 allows further insight into the mechanisms operating. It is significant 
that of the 45 individuals listed, 6 responded to $\mathrm{FG}$ and 42 responded to SG, but only 25 responded to $\mathrm{F} / \mathrm{S}$. This demonstrates active suppression of some response to SG by the addition of FG. This suppression is not always complete and halving of the response to $\mathrm{SG}$ in response to $\mathrm{F} / \mathrm{S}$ can also be taken as evidence of fast grower triggered suppression. This phenomenon appears in 3 out of 12 staff members, 9 out of 14 TT/BT patients, 7 out of 7 BL patients and 5 out of 12 LL patients. The mechanism of this suppression might be due to suppressor cells or suppressor complexes in which the antigens involved might be the group iii or the group iv antigens of fast growers. Our previous studies showing an almost normal distribution in responses of leprosy patients to some fast growing species ${ }^{6}$ containing group iii antigen shows that in most cases at least, this suppression must be triggered by the group iv (species specific) antigens of particular species, or by a unique presentation of group $\mathrm{i}$ antigens.

Responses to Burulin indicate recognition of groups i and/or ii antigens. These are strikingly missing from all patient groups, only 5 out of 33 patients responding, compared with 10 out of 12 staff members. This means that the responses shown by most leprosy patients to SG are directed against the group iv antigens of those slowly growing species that they have met.

Table 3 also gives results of five individuals (one staff member and four BL/LL patients), that we cannot yet explain with any confidence. These are marked in the table with asterisks.

In summary the majority of leprosy patients appear incapable of producing a positive skin-test response to groups i and ii antigen. They recognize slow growing species by response to their group iv (species specific) antigens and have suppressor mechanisms triggered by the group iv antigens of particular fast growing species. These phenomena are present in all the parts of the leprosy spectrum studied, although there is some variation between them. Whether the observed phenomena are a prerequisite for the development of disease, or a consequence of it, is difficult to answer. However, it may be of significance that some staff members also display the same phenomena. One tentative conclusion might be that ability to recognize group $\mathrm{i}$ antigen is crucial to the early detection and successful limitation of infectious challenge with the leprosy bacillus.

\section{Acknowledgements}

We would like to thank the patients and staff of Anandaban Leprosy Hospital for their co-operation and Lepra and the Leprosy Mission for their financial support.

\section{References}

1 Stanford JL, Grange JM. The meaning and structure of species as applied by Mycobacteria. Tubercle, $1974 ; 55: 143-152$. 
2 Shield MJ, Stanford JL, Paul RC, Carswell JW. Multiple skin-testing of tuberculosis patients with a range of new tuberculins and a comparison with leprosy and Mycobacterium ulcerans infection. Journal of Hygiene, 1977; 78, 331-348.

3 Stanford JL, Revill WDL, Gunthorpe WJ, Grange JM. The production and preliminary investigation of Burulin, a new skin-test reagent for Mycobacterium ulcerans infection. Journal of Hygiene, 1975;74, 7-16.

4 Stanford JL, Bahr GM, Nye PM, Rook GAW, Shield MJ. Common mycobacterial antigens may be important in leprosy. 1981, Proceedings of the Symposium on Mycobacterium leprae, Bombay February 1981, in press.

5 Stanford JL, Shield MJ, Rook GAW. An investigation of a range of new tuberculins. Part 1: The effects of aging, place of domicile, BCG immunisation and tuberculosis. Bulletin of the International Union against Tuberculosis, 1981, (in press).

6 Stanford JL, Shield MJ, Rook GAW. Mycobacterium leprae, other mycobacteria and a possible vaccine. Proceedings of the XI International Leprosy Congress, Mexico City. (International Congress Series No. 466) Amsterdam; Excerpta Medica. pp. 102-7, (1978). 